\title{
Future Perspective for Incorporation and Removal of Chemical Agents from Contaminated Surfaces ${ }^{\dagger}$
}

\author{
Răzvan Petre 1,*, Raluca Ginghină ${ }^{1}$, Liliana Rece ${ }^{1}$, Nicoleta Petrea ${ }^{1}$, Irina Elena Chican ${ }^{2}$ \\ and Florica Adriana Jerca ${ }^{3}$ \\ 1 Scientific Research Centre for CBRN Defence and Ecology, 225 Oltenitei Av., 041309 Bucharest, Romania; \\ ginghinaraluca@gmail.com (R.G.); lili_rece@yahoo.com (L.R.); nicoleta.petrea@yahoo.com (N.P.) \\ 2 The National Institute for Research \& Development in Chemistry and Petrochemistry, 202 Spl. \\ Independentei. 060021 Bucharest, Romania; irina_chican@yahoo.com \\ 3 Center for Organic Chemistry "Costin D. Nenitescu”, 202B Spl. Independentei, 060021 Bucharest, Romania; \\ adriana_jerca@yahoo.com \\ * Correspondence: petre.razvan@gmail.com \\ + Presented at the 15th International Symposium "Priorities of Chemistry for a Sustainable Development" \\ PRIOCHEM, Bucharest, Romania, 30th October-1st November 2019.
}

Published: 9 October 2019

Keywords: chemical decontamination; hydrogels; contaminants incorporation

Decontamination for military purposes represents the process of removing/neutralizing of chemical, biological, radiological and nuclear (CBRN) agents so that they no longer represent a risk for personnel. Current research and development (R\&D) is focused on developing a decontamination system/procedure that would overcome the limitation of classical systems (excessive labor and resource, massive water consumption, corrosive, toxic). In order to restore the combat effectiveness of equipment as soon as possible, we propose the decontamination solution with a water soluble hydrogel which will ensure the incorporation and removal of chemical agents from contaminated surfaces. The technical solution can be used to fix the contaminants in place in a safe manner until the operative situation allows the decontamination operations to be carried out [1].

The utilization is facile and it takes place in three simple steps: application, dry off and peel off. Another advantage represents the fact that can be used on a variety of surfaces: metal, concrete, wood, plastic, glass, etc.

Tests were performed according to the standard procedure on different support materials using nerve-type chemical warfare agents (CWA) simulant dimethyl methylphosphonate (DMMP) to the contamination norms stipulated in the NATO regulations. Different hydrogels with different compositions and drying times were used in order to study their ability to incorporate the contaminant.

The obtained results for the incorporation and removal of the chemical contaminants from the support materials were very good, achieving decontamination levels between $99.9-100 \%$.

In agreement with the obtained results, we conclude that the tested hydrogel formulas have great potential for future use in the incorporation and decontamination of surfaces contaminated with CWA, using simple and safe operations (Figure 1). 


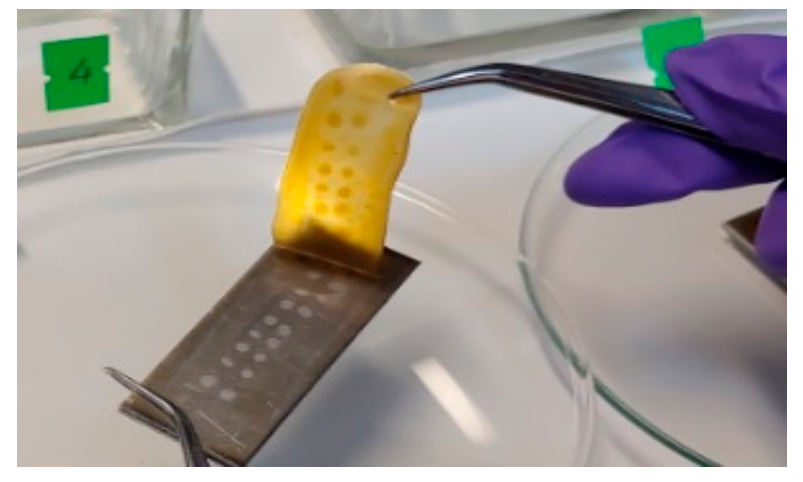

Figure 1. Decontamination of metal surface with hydrogel.

Acknowledgments: This work was supported by a grant of the Romanian National Authority for Scientific Research, CNDI-UEFISCDI, project number PN-III-P1-1.2-PCCDI, No. 70/2018.

\section{Reference}

1. Kalia, S. Polymeric Hydrogels as Smart Biomaterials; Springer International Publishing: Cham, Switzerland, 2016. 